\title{
ECG non-gated multi-detector computed tomography protocol prior to catheter ablation of atrial fibrillation provides sufficient data quality with lower radiation exposure compared to ECG-gated protocol. A prospective, randomized and blinded study
}

\author{
Zbynek Tudos ${ }^{\mathrm{a}}$, Tomas Skala ${ }^{\mathrm{b}}$, Martin Homolac, Ondrej Moravec ${ }^{\mathrm{b}}$, Milos Taborsky ${ }^{\mathrm{b}}$, Martin Kocher ${ }^{\mathrm{a}}$, Marie Cerna ${ }^{\mathrm{a}}$, \\ Filip Ctvrtlika, Frantisek Odstrcila,d, Katerina Langovaa ${ }^{e}$ Olga Klementova ${ }^{f}$
}

\begin{abstract}
Background. The role of ECG-gating in left atrium (LA) computed tomography (MDCT) imaging is not precisely defined. Methods and Results. 62 patients were randomized according to ECG gating with prospective evaluation of image quality, Volume CT Dose Index, Dose Length Product, Effective Dose and registration error between anatomical map and MDCT. We found significant difference in all radiation variables, but not in visual quality, registration error, $C A$ duration, CA fluoroscopy time and CA fluoroscopy dose.

Conclusion. Helical non-gated MDCT achieved a radiation dose more than four times lower with comparable image quality and course of ablation compared to ECG-gated protocol.

Key words: atrial fibrillation, catheter ablation, pulmonary veins, left atrium, multi-detector computed tomography, cardiac imaging

Received: December 21, 2017; Accepted: August 6, 2018; Available online: August 28, 2018 https://doi.org/10.5507/bp.2018.045

${ }^{a}$ Department of Radiology, Faculty of Medicine and Dentistry, Palacky University Olomouc and University Hospital Olomouc, Czech Republic ${ }^{b}$ Department of Internal Medicine I - Cardiology, Faculty of Medicine and Dentistry, Palacky University Olomouc and University Hospital Olomouc, Czech Republic

'Department of Medical Physics and Radiation Protection, University Hospital Olomouc, Czech Republic

${ }^{d}$ Department of Radiological Methods, Faculty of Health Sciences, Palacky University Olomouc, Czech Republic

${ }^{e}$ Department of Medical Biophysics, Faculty of Medicine and Dentistry, Palacky University Olomouc, Czech Republic

${ }^{f}$ Department of Anesthesiology and Intensive Care Medicine, Faculty of Medicine and Dentistry, Palacky University Olomouc and University Hospital Olomouc, Czech Republic

Corresponding author: Tomas Skala, e-mail: tomasskala@gmail.com
\end{abstract}

\section{INTRODUCTION}

Atrial fibrillation (AF) is the most common type of cardiac arrhythmia. Catheter ablation (CA) is an effective treatment for AF. The cornerstone of CA is pulmonary vein (PVs) isolation. A non-invasive visualization of PVs and the left atrium (LA) is used to assess the LA anatomy accurately in order to locate ablation lesions accurately and minimize complications. Currently, the most commonly used imaging method is multi-detector computed tomography (MDCT). Magnetic resonance imaging is used less commonly because of its lower spatial resolution, longer procedure and worse availability ${ }^{1}$. The goal of the MDCT examination is the creation of a 3D digital fusion of the MDCT data with a fast electroanatomic map (FAM). It is also used for a visual inspection of the anatomy when planning the intervention, e.g., when considering the use of balloon cryo-ablation. Using MDCT images maximizes efficacy, shortens duration and generally makes CA more accurate ${ }^{2-4}$. Although there is general agreement on the usefulness of MDCT, there is no consensus on the specific technical parameters of MDCT acquisition. One of the controversial questions is whether
ECG gating during MDCT data acquisition is absolutely necessary for CA purposes.

The aims of our study were to compare the data quality of MDCT images with and without ECG gating, to compare the radiation dose involved in both methods and to evaluate the effect of the quality of MDCT data on the ablation procedure.

\section{MATERIAL AND METHODS}

\section{Study design}

The study is designed as a prospective, randomized, blinded study with a predefined MDCT and ablation protocol. Patients indicated for AF ablation were randomized by a radiologist without any knowledge of the clinical status of the patients into two equal groups: group A underwent ECG-gated MDCT, group B underwent helical non-gated MDCT. Cardiac MDCT was performed 1-24 h before ablation. The study protocol was established prior to the enrollment of the first patient and was applied in all aspects to all patients. The team performing AF ablation was blinded with respect to the MDCT technique used 
from enrollment until the end of the 12-month follow-up period after ablation.

\section{Patient group}

Patients with paroxysmal AF planned for CA were prospectively recruited.

Inclusion criteria: Paroxysmal AF, at least three episodes of AF in the last six months, age over 18 years, sinus rhythm during CT data acquisition, signed informed consent to this study.

Exclusion criteria: Persistent, long-standing or permanent AF, structural heart disease, valvular heart disease or history of valve disease surgery, use of amiodarone in the last three months, LV EF $<35 \%$, pregnancy, breast feeding.

The study was approved by the Ethic Committee of the University Hospital Olomouc and the Faculty of Medicine and Dentistry, Palacky University Olomouc. All patients signed an informed consent to participation in the study.

A total of 62 consecutive patients were enrolled and randomized to two groups: group A (ECG-gated MDCT, $\mathrm{n}=31$ ) and group B (helical MDCT, no ECG synchronization, $n=31$ ). There was no statistically significant difference in gender, age and body mass index (BMI) between the groups (Table 1).

\section{MDCT procedure}

All participants underwent examination on a 64-slice LightSpeed VCT scanner (General Electrics, Milwaukee, USA). The ECG curve was monitored in all participants and all of them had regular sinus rhythm during the data acquisition. The range of the examination reached from the bifurcation of the trachea to the heart apex (Fig. 1).

Study group A underwent cardiac MDCT examination with retrospective ECG gating with a tube voltage of 120 $\mathrm{kV}$, pitch $0.2: 1$, collimation $64 \times 0.625 \mathrm{~mm}$, gantry rotation

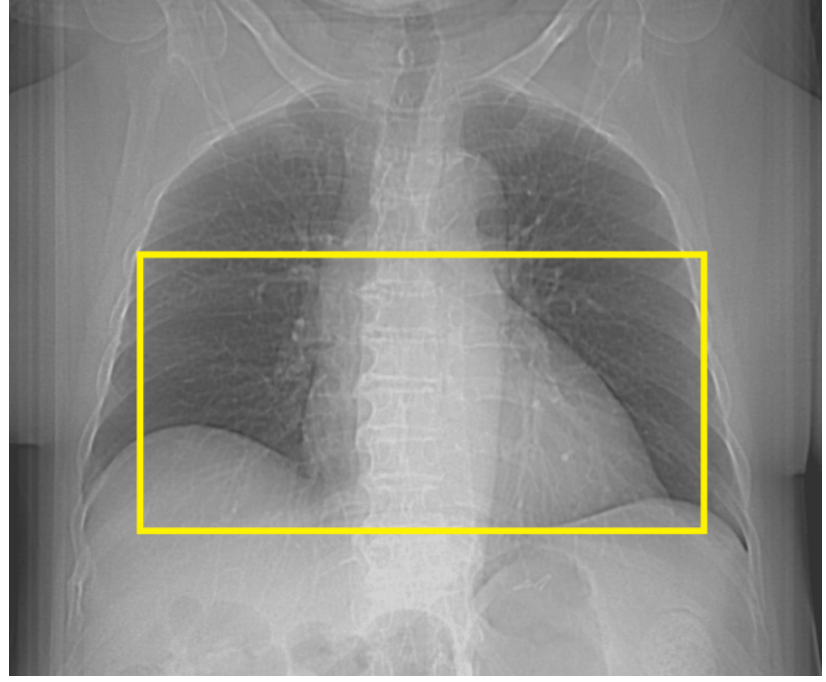

Fig. 1. Computed tomography scout; the yellow box shows the cranio-caudal extent of the CT examination.

$0.4 \mathrm{~s}$ and ECG-dependent tube current modulation as described previously 5 .

Study group B underwent helical MDCT without ECG gating with a tube voltage of $120 \mathrm{kV}$, pitch $0.984: 1$, collimation $64 \times 0.625 \mathrm{~mm}$ and gantry rotation $0.4 \mathrm{~s}$. Both the ECG-gated and helical non-gated MDCT examinations were performed with anatomically adapted tube current modulation based on attenuation profiles in the anteroposterior and lateral directions. The MDCT images were reconstructed with a slice thickness of $0.625 \mathrm{~mm}$ using the standard filtered back-projection method with kernel Standard. The participants in both groups received intravenously $70 \mathrm{~mL}$ of the iodinated contrast agent Iopromide (Ultravist $^{\circledR}$ 370, Bayer Healthcare, Berlin, Germany) with

Table 1. Demographic comparison of patients Groups A $(n=31)$ and B $(n=31)$.

\begin{tabular}{llrcc}
\hline \multicolumn{2}{l}{ Characteristics } & Group A & Group B & $P$ \\
\hline $\mathrm{n}$ & & 31 & 31 & 1.000 \\
Gender & males & 22 & 21 & 1.000 \\
& females & 9 & 10 & \\
\multirow{2}{*}{ Age } & median & 62 & 60 & 0.554 \\
& mean & 57.58 & 55.65 & \\
& SD & 12.47 & 12.27 & 0.116 \\
BMI & median & 26.53 & 29.28 & \\
& mean & 27.82 & 29.66 & \\
\hline
\end{tabular}

group A - retrospective ECG gated MDCT protocol

group B - helical non-gated MDCT protocol

$\mathrm{n}$ - number of subjects

BMI - Body Mass Index

$P$ - level of significance

SD - standard deviation 
a flow rate of $4 \mathrm{~mL} / \mathrm{s}$, followed by $30 \mathrm{~mL}$ of saline flush. The delay of data acquisition was triggered using the "bolus tracking" technique; the region of interest was located in the LA.

The MDCT images were routinely evaluated by a radiologist, who also performed the scoring of the quality of the visual data on a 5-point scale proposed by Wagner et al. (Table 2) $\left(\right.$ ref. $\left.^{6}\right)$.

\section{MDCT dosimetry}

The values of the volume computed tomography dose index $\left(\mathrm{CTDI}_{\mathrm{vol}}\right)$ and dose length product (DLP) were recorded from the MDCT scanner dose report, which is calculated and archived automatically. Subsequently, simple effective dose (ED) estimation was calculated according to the European Guidelines on Quality Criteria European Guidelines for MDCT by multiplying the DLP by the region-specific normalized effective dose, which is $0.017 \mathrm{mSv}^{*} \mathrm{mGy}^{1 *} \mathrm{~cm}^{-1}$ for chest CT (ref. ${ }^{7}$ ).

\section{Catheter ablation}

The staff members performing the intervention were fully blinded with respect to which CT technique was used in a particular patient. The study protocol was established prior to the enrollment of the first patient and was applied in all aspects to all patients. Ablation was performed under general anesthesia in all patients. Two sheaths were introduced via the femoral vein for an intracardiac echocardiography probe (AcuNav ultrasound catheter, Siemens Healthineers, USA) and decapolar diagnostic coronary sinus catheter (Inquiry ${ }^{\mathrm{TM}}$, St. Jude Medical, MN, USA). Two steerable transseptal sheaths (Agilis ${ }^{\mathrm{TM}}$
NxT Steerable Introducer, St. Jude Medical, MN, USA) were introduced via the femoral vein. A double transseptal puncture was performed in all patients. After the transseptal puncture a 3D electroanatomic map was created using Carto3 ${ }^{\mathrm{TM}}$ (Biosense Webster, CA, USA). A fast anatomical map (FAM) was created consistently in all patients (Fig. 2). The anatomical map was merged with a 3D model of LA using the CartoMerge ${ }^{\text {TM }}$ technology. A point-by-point radiofrequency wide-antral ablation was performed in all patients using a Navistar ${ }^{\mathrm{TM}}$ ablation catheter (Biosense Webster, CA, USA) to achieve isolation of the ipsilateral pulmonary veins (PVs) (Fig. 3). The ablation energy was set to $25-30 \mathrm{~W}$ in all patients, with a cool flow of $20 \mathrm{~mL} / \mathrm{min}$. The inter-lesion distance was intended to be less than $6 \mathrm{~mm}$. A Lasso ${ }^{\mathrm{TM}}$ catheter (Biosense Webster, CA, USA) was used to validate the isolation of the PVs (entry block) in all patients. In the event of ongoing AF prior to the final check for the isolation of PVs a direct current cardioversion was performed. Extrapulmonary ablation was not performed unless typical atrial flutter occurred or was documented before ablation or a spontaneous procedural atrial tachycardia occurred. Mapping and ablation of these tachycardias was allowed. No inducibility testing or provocation of extrapulmonary triggers was performed after the isolation of the PVs. After the isolation of the PVs we waited for 30 min and then adenosine was applied intravenously to test dormant conduction using a double lasso technique. At least $18 \mathrm{mg}$ of adenosine was used for the test. The dose was considered sufficient if it resulted in a second- or third-degree atrioventricular block. Additional ablation was performed in those PVs with conduction recovery af-

Table 2. Visual data quality on 5-point scale.

\begin{tabular}{ll}
\hline 1 & excellent depiction of the LA with smooth endocardial surface \\
2 & good depiction of the LA with only discrete irregularities of the endocardial surface \\
3 & moderate depiction of the LA with moderate irregularities of the endocardial surface \\
4 & poor depiction of the LA with severe irregularities of the endocardial surface \\
5 & segmentation of the LA failed \\
\hline
\end{tabular}

LA left atrium

Table 3. Number of subject according to visual image quality.

\begin{tabular}{lcc}
\hline Degree of depiction of the endocardial surface of the LA & Group A & Group B \\
\hline excellent (1) & 14 & 6 \\
good (2) & 12 & 20 \\
moderate (3) & 3 & 5 \\
poor (4) & 2 & 0 \\
failed (5) & 0 & 0 \\
\hline
\end{tabular}


ter the adenosine testing. To determine whether dormant conduction had been eliminated after re-ablation, another round of adenosine testing was performed. No specific waiting time was required before additional adenosine tests after reablation for reconduction. Once all the PVs with conduction recovery were re-isolated, the procedure was ended. The overall duration of the ablation procedure, fluoroscopy time and fluoroscopy dose were recorded at the end of the procedure. The automatic calculation of the registration error, i.e., the average distance between the respective points of the left atrium surface on the 3D MDCT model and FAM, was documented.

\section{Statistics}

The following parameters were selected for statistical comparison between the groups: visual data quality, $\mathrm{CTDI}_{\mathrm{vol}}$, DLP, effective dose of MDCT, mean registration error, duration of CA procedure, fluoroscopy time and dose during CA. Tests of normality (the Shapiro-Wilk test) revealed non-normal distribution of the data. The data were expressed as median, means and standard deviation (SD). The differences between the two independent groups were analyzed by means of the Fisher exact test and the Mann-Whitney U-test. Values with $P<0.05$ were considered statistically significant. All statistical analyses were conducted with IBM SPSS Statistics 23 (IBM Corporation, 2015).

\section{RESULTS}

All patients underwent the MDCT examination successfully. All examinations were appropriate for the subsequent ablation procedure.

\section{Visual quality of CT model of left atrium}

No statistically significant difference in the visual quality of the MDCT images was found; in both groups the mean and median were around 2, i.e., discrete irregularities of the endocardial surface (Table 3 and $4 \mathrm{a}$ ).

\section{Dosimetry}

A comparison of the dosimetric variables demonstrated differences in CTDI $_{\mathrm{vol}}$, DLP and ED between the groups (mean values $89.6 \pm 6 \mathrm{mGy}, 1438.9 \pm 147.8$ $\mathrm{mGy}{ }^{*} \mathrm{~cm}, 24.46 \pm 2.51 \mathrm{mSv}$ in group A versus $19.2 \pm 4.3$ $\mathrm{mGy}, 328.2 \pm 73.8 \mathrm{mGy}^{*} \mathrm{~cm}, 5.58 \pm 1.26 \mathrm{mSv}$ in group B); these differences were statistically significant on the level $P<0.0001$ (Table 4a).

\section{Catheter ablation}

PV isolation (entry block) was achieved in all patients. The elimination of dormant conduction after the adenosine test was achieved in all PVs. There was no statistically significant difference between the groups in any of the parameters that were monitored, i.e., the duration of the procedure, fluoroscopy time, fluoroscopy dose and registration error (Table 4b).

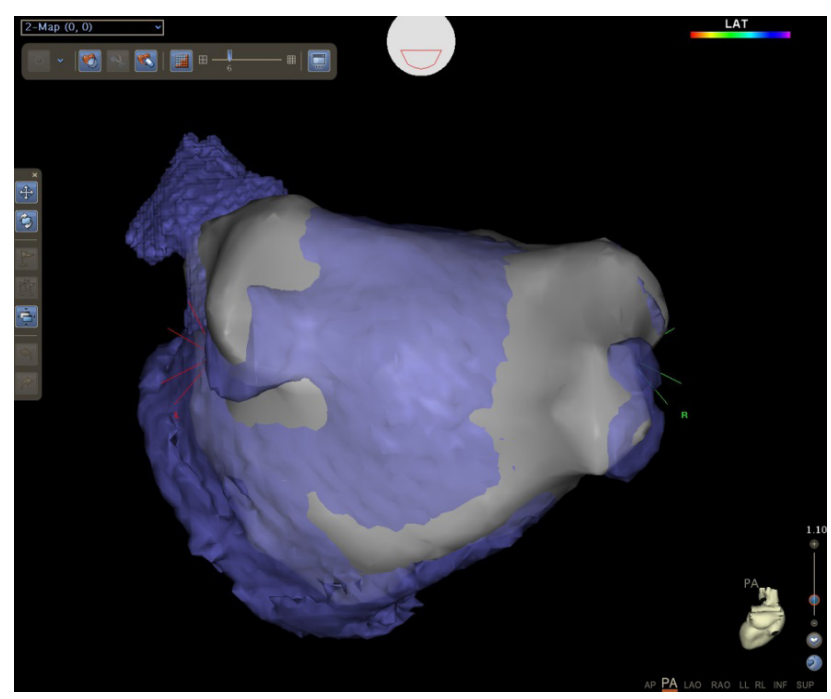

Fig. 2. Merge of left atrium maps: the blue map is the 3D MDCT model, the gray map is the electro-anatomic map.

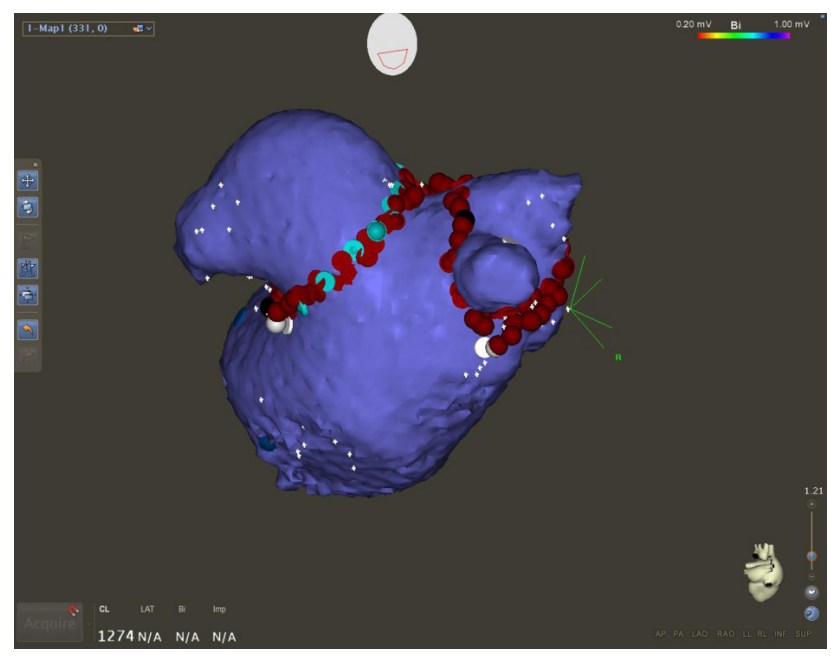

Fig. 3. CARTO3 CT map of the left atrium: red spots denote points of application of radiofrequency energy, white spots denote anchors of the electro-anatomic map (anatomical landmarks defined by intra-cardial ultrasound), pale blue spots denote points planned for ablation, dark blue spots denote mitral anulus, black spot denotes point of isolation of pulmonary vein.

\section{DISCUSSION}

The MDCT of LA and PVs represents a standard part of pre-procedure planning in many ablation centers, because the fusion of 3D CT data and an electroanatomic map seems to maximize efficacy, shorten the duration and generally make AF ablation more accurate ${ }^{2-4}$. Having MDCT data available is certainly not absolutely necessary, especially when using intracardiac echocardiography (ICE). However, ICE is not used in most ablation centers and not all patients have good ICE image quality. In such cases, the 3D CT model can allow precise targeting of the ablation lesion. 3D CT models of LA are used in 
Table 4. MDCT and catheter ablation parameters.

\begin{tabular}{|c|c|c|c|c|}
\hline a) MDCT & & Group A & Group B & $P$ \\
\hline \multirow[t]{3}{*}{ Visual quality on 5-point scale } & median & 2 & 2 & 0.102 \\
\hline & mean & 1.77 & 2.00 & \\
\hline & SD & 0.88 & 0.63 & \\
\hline \multirow[t]{3}{*}{$\mathrm{CTDI}_{\mathrm{vol}}(\mathrm{mGy})$} & median & 88.06 & 20.22 & $<0.0001$ \\
\hline & mean & 89.55 & 19.19 & \\
\hline & SD & 5.99 & 4.33 & \\
\hline \multirow[t]{3}{*}{$\mathrm{DLP}\left(\mathrm{mGy}{ }^{*} \mathrm{~cm}\right)$} & median & 1407.49 & 334.85 & $<0.0001$ \\
\hline & mean & 1438.87 & 328.28 & \\
\hline & $\mathrm{SD}$ & 147.75 & 73.83 & \\
\hline \multirow[t]{3}{*}{ Effective dose (mSv) } & median & 23.93 & 5.69 & $<0.0001$ \\
\hline & mean & 24.46 & 5.58 & \\
\hline & SD & 2.51 & 1.26 & \\
\hline b) Catheter ablation & & Group A & Group B & $P$ \\
\hline \multirow[t]{3}{*}{ registration error $(\mathrm{mm})$} & median & 2.38 & 2.37 & 0.612 \\
\hline & mean & 2.42 & 2.43 & \\
\hline & $\mathrm{SD}$ & 0.72 & 0.46 & \\
\hline \multirow[t]{3}{*}{ Procedure time $(\min )$} & median & 120 & 120 & 0.108 \\
\hline & mean & 131.61 & 119.84 & \\
\hline & SD & 32.57 & 33.18 & \\
\hline \multirow[t]{3}{*}{ Fluoroscopy time (min) } & median & 4.32 & 3.33 & 0.251 \\
\hline & mean & 4.48 & 3.89 & \\
\hline & SD & 2.19 & 1.83 & \\
\hline \multirow[t]{3}{*}{ Fluoroscopy dose $\left(\mathrm{Gy}^{*} \mathrm{~cm}^{2}\right)$} & median & 3.02 & 3.28 & 0.735 \\
\hline & mean & 3.99 & 3.91 & \\
\hline & SD & 2.79 & 2.91 & \\
\hline
\end{tabular}

group A - retrospective ECG gated MDCT protocol

group B - helical non-gated MDCT protocol

$\mathrm{CTDI}_{\mathrm{vol}}$ - volume computed tomography dose index

DLP - dose length product

$P$ - level of significance, statistically significant result are bold

many centers around the world and are very likely to be used more in the future. Although there is general agreement on the usefulness of MDCT imaging, there is no broad consensus on the specific technical parameters of acquiring CT images. One of the unclear questions remains whether ECG synchronization is absolutely necessary for CA.

Since MDCT is the significant source of radiation, there is a strong effort to optimize the MDCT protocol so that the resulting MDCT images are of sufficient quality and the radiation dose is the lowest possible. Endeavoring to reduce the radiation dose is very important as another dose of radiation is applied to the patient because of the fluoroscopy during CA; furthermore, in the event of a recurrence of AF, a new MDCT is often obtained. Another MDCT is sometimes used to assess complications after CA.
One of the aspects affecting MDCT image quality and the radiation dose is the use of the synchronization of data acquisition with an ECG curve in the MDCT scanner. The main technical difference affecting the radiation dose between ECG-gated MDCT and helical non-gated MDCT is the value of the pitch, which is defined as the distance that the table travels per complete rotation of the gantry divided by the detector width. For helical MDCT scanning, a higher pitch can be used, whereas for ECGgated MDCT a lower pitch is necessary in order to provide partially overlapping MDCT projections that allow the data from different phases of the cardiac cycle to be sorted $^{8}$. Thus, the pitch is inversely proportional to the resulting radiation dose. It is absolutely essential to use ECG gating to perform CT coronarography, but the question is whether this approach is also unavoidable in the MDCT of LA and PVs. Besides the retrospective ECG 


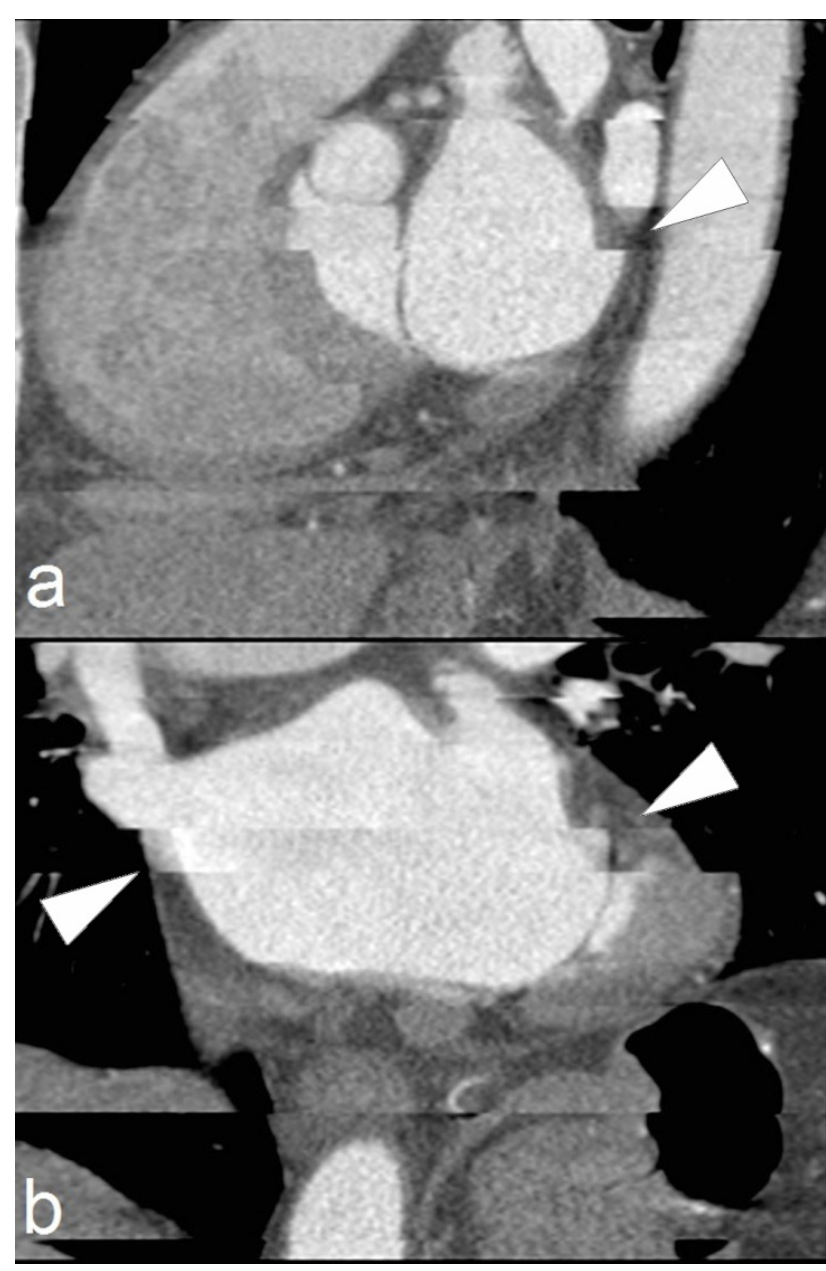

Fig. 4. Retrospectively ECG-gated MDCT in a) the sagittal and b) the coronal plane; the images show stair-step artifacts on the surface of the left atrium (arrowheads) resulting from incorrect data registration; the visual image quality was graded as "poor".

gating, it is also possible to use prospective ECG triggering (also known as the "step-and-shoot" technique), in which the data acquisition is limited only to the diastole, thus reducing the radiation dose, but other phases of the cardiac circle are not available for post-processing ${ }^{9}$. Both prospectively ECG-triggered and retrospectively ECGgated techniques can be compromised by the presence of stair-step artifacts significantly reducing the image quality of the MDCT model ${ }^{8,10,11}$. In our work, we strictly used retrospective $\mathrm{ECG}$ gating.

The arguments for ECG synchronization are: fewer motion artifacts of the LA contours and the ostia of the PVs, the possibility of observing the movement of the LA wall and changes of diameter in PVs during the heart cycle or the choice to select the optimal phase of the heart cycle for the subsequent fusion.

The argument against ECG synchronization is that non-synchronized MDCT images may be blurred as a result of motion, and yet they are fully usable for both anatomical visual evaluation and subsequent digital fusion. Furthermore, it is possible to question the importance of displaying the LA volume and changes in the diameter of the pulmonary veins. These changes are measurable but

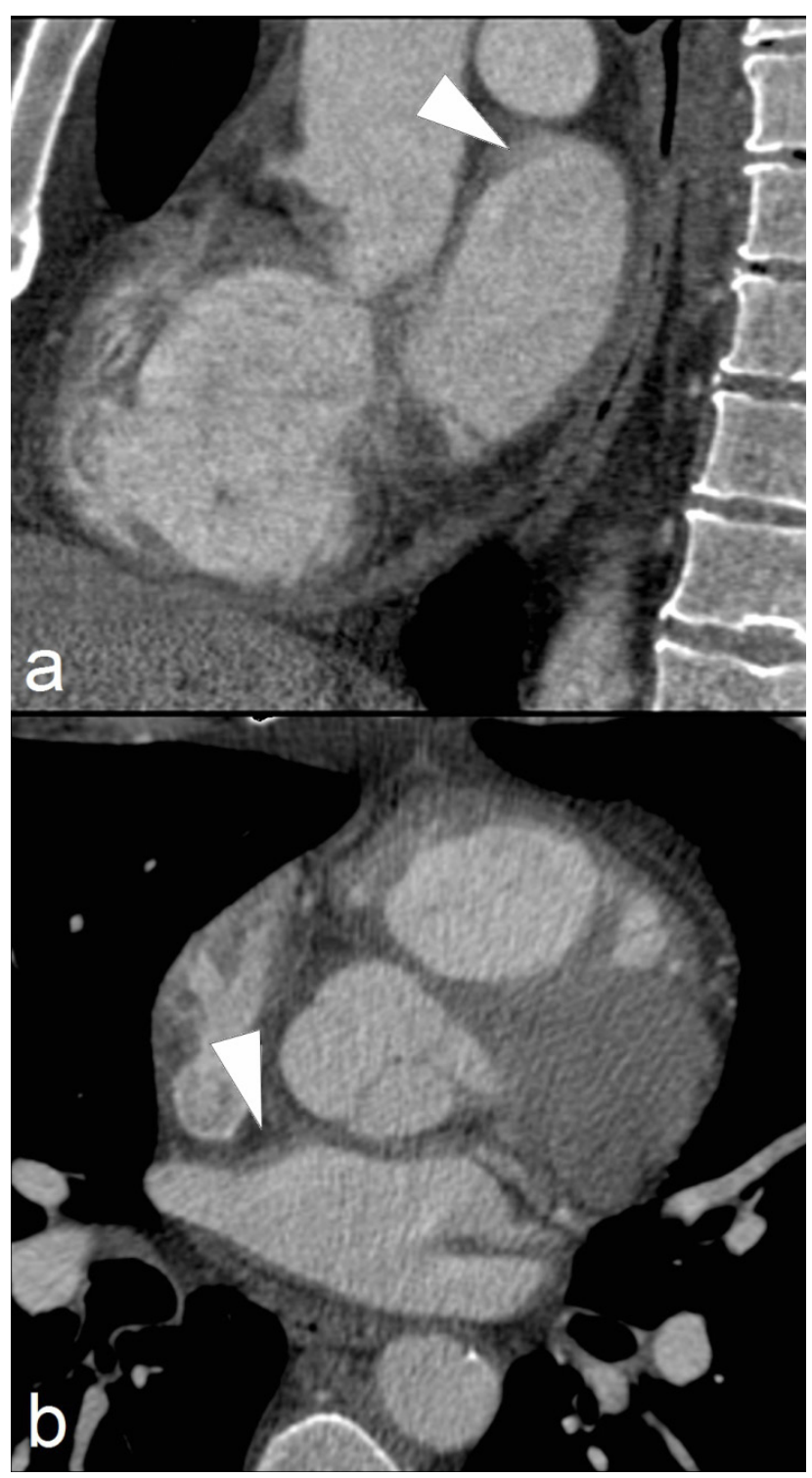

Fig. 5. Helical non-gated MDCT in a) the sagittal and b) the axial plane; the images show blurred surface of the left atrium (arrowheads) as a result of motion; the visual image quality was graded as "moderate".

they are likely to be negligible with regard to the ablation performance because ablation systems do not allow ablation to be limited to a particular phase of the heart cycle and therefore a certain inaccuracy is always present even when using best-quality ECG-gated MDCT data.

So far, two papers have been published that directly compared image quality and the radiation dose of ECGgated and helical non-gated MDCT protocols. However, both papers were retrospective, one of them included only a limited number of subjects and, in particular, neither of these two papers also evaluated the effect of the MDCT technique on the performance, parameters and results of subsequent CA (ref. ${ }^{6,9}$ ).

The aims of our study were to provide a prospective, randomized, blinded comparison of MDCT image quality between the ECG-gated and helical non-gated techniques and a comparison of the radiation dose between the two 
methods, as well as an evaluation of the effect of MDCT data quality on the ablation process since most centers prefer the MDCT of LA using ECG gating ${ }^{12-18}$.

The main argument for the use of ECG gating is the better visual quality of the LA MDCT model. The results of our work are in disagreement with this premise. In group A (ECG-gated MDCT), the average visual quality score was 1.77 points out of five. A total of 26 out of 31 subjects were placed in the "perfect" or "good" category. Low-score ECG-gated images were compromised by stairstep artifacts as a result of cardiac motion or the patient's non-compliance with the instruction to hold their breath (Fig. 4); three subjects were rated "moderate" and two "poor". In group B (helical non-gated MDCT), the average visual quality score was two points out of five. A total of 26 out of 31 subjects achieved "perfect" or "good" quality. Five patients showed blurred LA contours as a result of motion and were rated as "moderate" (Fig. 5). Thus, our work does not confirm the assumption that ECG-gated MDCT data possess a better visual quality. The comparable visual quality of the data between the two techniques was previously published by other teams ${ }^{6,9}$. Moreover, the blurring of the LA contours is closer to the real-world reality of a beating heart than a sharp stair-step artifact.

The evaluation of the visual quality of CT images is subjective, so, in addition to visual quality, we also used an objective measurement of a match between the 3D MDCT model surface and the FAM of LA. This deviation (also called the registration error), can be expressed simply as the mean distance between the corresponding points in the CT model and the FAM measured in millimeters. We did not find a statistically significant difference between the mean registration error between group A and group B (2.42 mm vs. $2.43 \mathrm{~mm})$, which is also consistent with previous research ${ }^{6}$. In other words, for the physician performing the intervention, it was completely insignificant which MDCT technique was used; the fit between the 3D MDCT model and the FAM was the same.

Complete isolation of the PVs was achieved in all patients. There was no positive effect of ECG gating on the duration of the ablation procedure; the duration of $\mathrm{CA}$ was even 14 minutes shorter in the patients with helical non-gated MDCT, but this difference was not statistically significant. A similar situation occurred in the comparison of the fluoroscopy time during CA - in group B the fluoroscopy time was insignificantly shorter than in group A. Therefore, our research did not demonstrate any positive effect of ECG gating on the performance and immediate result of $\mathrm{CA}$.

Another crucial aim of our study was to compare the radiation dose between ECG-gated and helical non-gated MDCT. The values of CTDI ${ }_{\text {vol }}$ and DPL were chosen for the comparison. From the point of view of the MDCT technique, the two variables are more suitable for comparing different MDCT protocols than ED because they are better-defined quantities and their calculation is not influenced by the methodology that is selected. For both CTDI $_{\text {vol }}$ and DPL, a significantly higher value was found for ECG-gated MDCT. Both values were about 4.5 times higher, which is directly projected into the subsequently calculated ED estimations. The cause of this multiple difference is undoubtedly the different values of the pitch, which is inversely proportional to the resulting CTDI and DLP. The difference in the values of the pitch for ECG-gated MDCT (0.2:1) and helical non-gated MDCT (0.984:1) apparently inversely approximates the difference between the average values of CTDI ${ }_{\text {vol }}$ and DLP. Our DLP and ED values of the helical non-gated MDCT were comparable to those reported in the previous article ${ }^{6}$; for ECG-gated MDCT, the mean values were relatively higher, probably because we used a protocol originally intended for CT coronarography. If, however, we compare the observed ED values with other papers using 64-row scanners for retrospectively ECG-gated cardiac MDCT, the ED values are comparable ${ }^{19}$. The comparison of ED between different studies may be complicated because it is only an estimation, which may vary considerably according to the methodology or software used. The easiest way to estimate ED is to multiply DLP by an organ-specific coefficient. We used the commonly used chest CT coefficient, i.e., $0.017 \mathrm{mSv}{ }^{*} \mathrm{mGy}-1{ }^{*} \mathrm{~cm}-1$ (ref. ${ }^{7,20}$ ), but there are also different recommendations for chest CT, e.g., 0.019 $\mathrm{mSv} * \mathrm{mGy}-1{ }^{*} \mathrm{~cm}-1\left(\right.$ ref. $\left.^{21}\right)$. Additionally, there are works recommending a coefficient of $0.028 \mathrm{mSv} * \mathrm{mGy}-1 * \mathrm{~cm}$ 1 for cardiac MDCT $\left(\right.$ ref. $^{22}$ ); in this respect the ED values can be systematically underestimated.

Besides the value of the pitch, the radiation dose of MDCT may also be affected by other parameters, so it is necessary to find a suitable compromise between the technical quality of the MDCT images and the resulting radiation dose. In addition to, e.g., slice thickness, anatomically adapted tube current modulation or the cranio-caudal extent of the examination, modern MDCT scanners offer modern image reconstruction algorithms. Compared to the traditionally used filtered back-projection technique, contemporary scanners have the option of iterative reconstruction algorithms, such as adaptive statistical iterative reconstruction. These techniques reduce the noise in the CT image and thus allow reduction of the voltage and current values of the X-ray tube. In recent years, more advanced iterative techniques (e.g., model-based iterative reconstruction) have become available; their long calculation time is a disadvantage, but they can reduce the ED to below $1 \mathrm{mSv}$ (ref. ${ }^{23}$ ). ECG-gated scan dose reduction can be also achieved by using ECG-controlled tube current modulation technice (also referred to as "ECG pulsing"). This algorithm modulates tube current according to a cardiac cycle phases as cardiac motion is greatest during systole and least during diastole, the image data is most likely sharpest during diastole. Accordingly, this algorithm reduces the tube current by $75 \%$ during systole, in which image reconstruction is not likely to be of interest ${ }^{5}$

\section{Study limitations}

Compared to previously published studies, the prospective character of the study, with standard randomization and double blindness, is among the merits of our work. Randomization was performed by a radiologist 
without knowledge of the patient's health condition. The entire intervention team remained blinded to the MDCT technique used. Furthermore, our study group was larger than that of Weber et al. ${ }^{6}$ and more homogeneous than the group of Thai et al. and also exceeds the number of subjects in several subgroups ${ }^{9}$. It also contains a direct comparison of the effects of two MDCT techniques on subsequent CA. As a possible disadvantage, it must be admitted that the trend for research in cardiac imaging is currently towards 320-row or dual-source scanners. However, 64-row and 128-row scanners, which offer a choice between ECG-gated and helical non-gated techniques, are still widely used for CA planning in clinical practice. The choice between high-pitch helical technology and ECG-triggering/gating is also relevant in dualsource MDCT scanners ${ }^{9,24}$. Thus, our research is less relevant only to 320-row MDCT scanners with volume data acquisition.

Limitations may include the use of a protocol originally designed for CT coronarography, where much greater detail is needed than with LA imaging. It would be possible to find better compromise between signal-to-noise value, spatial resolution and radiation dose for LA imaging in clinical practice. Theoretically, it might be more appropriate to use prospective ECG triggering, as it is likely that prospective triggering would achieve a lower ED than retrospective gating. However, the main goal of our study was to confirm that helical non-gated MDCT is a fully adequate method with comparable image quality and CA outcomes, regardless of which ECG synchronization technique it is compared to.

\section{CONCLUSIONS}

Helical non-gated multi-detector computed tomography of the left atrium and pulmonary veins achieved a radiation dose more than four times lower with comparable visual image quality, registration error between the 3D MDCT model and FAM and the course and result of catheter ablation compared to ECG-gated multi-detector computed tomography.

\section{ABREVIATIONS}

AF, Atrial fibrillation; CA, Catheter ablation; CTDI volume computed tomography dose index; DLP, Dose length product; ED, Effective dose; FAM, Fast electroanatomic map; LA, Left atrium; MDCT, Multi-detector computed tomography; PV, Pulmonary vein.

Acknowledgment: Supported by Palacky University grant IGA_LF_2018_002.

Author contributions: ZT: study design, data analysis, data interpretation, manuscript writing and literature search; TS: study design, data analysis, data interpretation, manuscript writing and literature search; $\mathrm{MH}$ : data collection, data interpretation; OM, FO: data collection; MT, MK: final approval of the manuscript; $\mathrm{MC}, \mathrm{FC}, \mathrm{OK}$ : data in- terpretation, critical revision of the manuscript; KL: statistical analysis.

Conflict of interest statement: None declared.

\section{REFERENCES}

1. Hamdan A, Charalampos K, Roettgen R, Wellnhofer E, Gebker R, Paetsch I, Jahnke C, Schnackenburg B, Tang M, Gerds-Li H, Fleck E. Magnetic Resonance Imaging Versus Computed Tomography for Characterization of Pulmonary Vein Morphology Before Radiofrequency Catheter Ablation of Atrial Fibrillation. Am J Cardiol 2009;104(11):1540-6.

2. Bertaglia E, Bella PD, Tondo C, Proclemer A, Bottoni N, De Ponti R, Landolina M, Bongiorni MG, Coro L, Stabile G, Russo AD, Verlato R, Mantica M, Zoppo F. Image integration increases efficacy of paroxysmal atrial fibrillation catheter ablation: results from the CartoMerge $^{\mathrm{TM}}$ Italian Registry. EP Eur 2009;11(8):1004-10.

3. Della Bella P, Fassini G, Cireddu M, Riva S, Carbucicchio C, Giraldi F, Maccabelli G, Trevisi N, Moltrasio M, Pepi M, Galli CA, Andreini D, Ballerini G, Pontone G. Image integration-guided catheter ablation of atrial fibrillation: a prospective randomized study. J Cardiovasc Electrophysiol 2009;20(3):258-65.

4. Martinek M, Nesser H-J, Aichinger J, Boehm G, Purerfellner H. Impact of integration of multislice computed tomography imaging into three-dimensional electroanatomic mapping on clinical outcomes, safety, and efficacy using radiofrequency ablation for atrial fibrillation. Pacing Clin Electrophysiol PACE 2007;30(10):1215-23.

5. Hermann F, Martinoff S, Meyer T, Hadamitzky M, Jiang C, Hendrich E, Hausleiter J. Reduction of radiation dose estimates in cardiac 64-slice CT angiography in patients after coronary artery bypass graft surgery. Invest Radiol 2008;43(4):253-60.

6. Wagner M, Butler C, Rief M, Beling M, Durmus T, Huppertz A, Voigt A, Baumann G, Hamm B, Lembcke A, Vogtmann T. Comparison of non-gated vs. electrocardiogram-gated 64-detector-row computed tomography for integrated electroanatomic mapping in patients undergoing pulmonary vein isolation. Europace 2010;12(8):1090-7.

7. Bongartz G, Golding SJ, Jurik AG, Leonardi M, van Persijn van Meerten E, Geleijns J, Jessen K.-, Panzer W, Shrimpton PC, Tosi G. European Guidelines on Quality CriteriaEuropean Guidelines for Multislice Computed Tomography. 1999;at <http://www.drs.dk/ guidelines/ct/quality/mainindex.htm >.

8. Desjardins B, Kazerooni EA. ECG-Gated Cardiac CT. Am J Roentgenol 2004;182(4):993-1010.

9. Thai W, Wai B, Lin K, Cheng T, Heist EK, Hoffmann U, Singh JP, Truong QA. Pulmonary Venous Anatomy Imaging With LowDose, Prospectively ECG-Triggered, High-Pitch 128-Slice DualSource Computed TomographyClinical Perspective. Circ Arrhythm Electrophysiol 2012;5(3):521-30.

10. Husmann L, Valenta I, Gaemperli O, Adda O, Treyer V, Wyss CA, VeitHaibach P, Tatsugami F, Schulthess V, K G, Kaufmann PA. Feasibility of low-dose coronary CT angiography: first experience with prospective ECG-gating. Eur Heart J 2008;29(2):191-7.

11. Machida H, Tanaka I, Fukui R, Shen Y, Ishikawa T, Tate E, Ueno E. Current and Novel Imaging Techniques in Coronary CT. RadioGraphics 2015;35(4):991-1010.

12. Cronin P, Kelly AM, Desjardins B, Patel S, Gross BH, Kazerooni EA, Morady F, Oral H, Carlos RC. Normative Analysis of Pulmonary Vein Drainage Patterns on Multidetector CT With Measurements of Pulmonary Vein Ostial Diameter and Distance to First Bifurcation. Acad Radiol 2007;14(2):178-88.

13. Manghat NE, Mathias HC, Kakani N, Hamilton MCK, MorganHughes G, Roobottom CA. Pulmonary venous evaluation using electrocardiogram-gated 64-detector row cardiac CT. Br J Radiol 2012;85(1015):965-71.

14. Choi SI, Seo JB, Choi SH, Lee S-H, Do K-H, Ko SM, Lee JS, Song J-W, Song K-S, Choi K-J, Kim Y-H, Lim T-H. Variation of the size of pulmonary venous ostia during the cardiac cycle: optimal reconstruction window at ECG-gated multi-detector row CT. Eur Radiol 2005;15(7):1441-5.

15. Avelar E, Durst R, Rosito GA, Thangaroopan M, Kumar S, Tournoux F, Chan RC, Hung J, Hoffmann U, Abbara S, Brady T, Cury RC. Comparison of the accuracy of multidetector computed tomog- 
raphy versus two-dimensional echocardiography to measure left atrial volume. Am J Cardiol 2010;106(1):104-9.

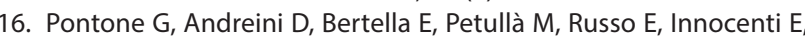
Mushtaq S, Gripari P, Loguercio M, Segurini C, Baggiano A, Conte E, Beltrama V, Annoni A, Formenti A, Guaricci Al, Casella M, Fassini G, Giovannardi M, Veglia F, Tondo C, Pepi M. Comparison of cardiac computed tomography versus cardiac magnetic resonance for characterization of left atrium anatomy before radiofrequency catheter ablation of atrial fibrillation. Int J Cardiol 2015;179:114-21.

17. Neumann A, Bohata S, Kerkovsky M, Pavlik T. Effect of the presence of calcium in the coronary arteries to the cardiac function. Ceska Radiol 2011;65(4):279-88.

18. Fahlenkamp UL, Lembcke A, Roesler R, Schwenke C, Huppertz A Streitparth F, Taupitz M, Hamm B, Wagner M. ECG-gated imaging of the left atrium and pulmonary veins: Intra-individual comparison of CTA and MRA. Clin Radiol 2013;68(10):1059-64.

19. Sun Z. Coronary CT angiography with prospective ECG-triggering: an effective alternative to invasive coronary angiography. Cardiovasc Diagn Ther 2012;2(1):28-37.

20. Huda W, Ogden KM, Khorasani MR. Converting Dose-Length Product to Effective Dose at CT. Radiology 2008;248(3):995-1003.
21. Bongartz G, Golding SJ, Jurik AG, Leonardi $M$, van Persijn van Meerten E, Rodriguez R, Schneider K, Calzado A, Geleijns J, Jessen K.-, Panzer W, Shrimpton PC, Tosi G. European Guidelines for Multislice Computed Tomography. 2004;at <http://www.msct.eu/ CT_Quality_Criteria.htm\#Download\%20the\%202004\%20CT\%20 Quality\%20Criteria>.

22. Gosling O, Loader R, Venables P, Rowles N, Morgan-Hughes G, Roobottom C. Cardiac CT: are we underestimating the dose? A radiation dose study utilizing the 2007 ICRP tissue weighting factors and a cardiac specific scan volume. Clin Radiol 2010;65(12):1013-7.

23. Annoni $A D$, Andreini $D$, Pontone $G$, Formenti $A$, Petullà $M$, Consiglio $E$, Nobili E, Baggiano A, Conte E, Mushtaq S, Bertella E, Billi F, Bartorelli AL, Montorsi P, Pepi M. Ultra-low-dose CT for left atrium and pulmonary veins imaging using new model-based iterative reconstruction algorithm. Eur Heart J Cardiovasc Imaging 2015;16(12):1366-73.

24. Iwayama T, Arimoto T, Ishigaki D, Hashimoto N, Kumagai YU, Koyama YO, Kiribayashi N, Netsu S, Nishiyama S, Takahashi H, Shishido T, Miyamoto T, Sato T, Watanabe T, Kubota I. The Clinical Value of Nongated Dual-Source Computed Tomography in Atrial Fibrillation Catheter Ablation. J Cardiovasc Electrophysiol 2016;27(1):34-40. 